\title{
Applications of the theory of evolution equations to general relativity
}

\author{
Alan D. Rendall \\ Max-Planck-Institut für Gravitationsphysik \\ Am Mühlenberg 1, 14476 Golm, Germany
}

\begin{abstract}
The theory of evolution equations has been applied in various ways in general relativity. Following some general considerations about this, some illustrative examples of the use of ordinary differential equations in general relativity are presented. After this recent applications of Fuchsian equations are described, with particular attention to work on the structure of singularities of solutions of the Einstein equations coupled to a massless scalar field. Next the relations between analytical and numerical studies of the Einstein equations are discussed. Finally an attempt is made to identify fruitful directions for future research within the analytic approach to the study of the Einstein equations.
\end{abstract}

\section{Introduction}

When general relativity is applied to model a physical system a connection must be made on some level between solutions of the Einstein equations, in general coupled to suitable matter fields, and the behaviour of the system concerned. As a consequence, a natural goal in general relativity is to obtain an overview of solutions of the Einstein equations which is as good as possible according to the criteria of generality, reliability and physical relevance. There are four main approaches to this goal which may be called algebraic, heuristic, numerical and analytical. In this article attention will be centred on the fourth of these but this subject will first be put in context by discussing the classification just indicated.

The approach referred to here as 'algebraic' is what is more commonly known as the field of 'exact solutions'. A reasonable strategy when confronted with unfamiliar differential equations is to look for solutions in terms of elementary functions. The basic work to be done consists of algebraic manipulations, hence the terminology chosen. For sufficiently complicated equations (e.g. the Einstein equations) the solutions obtained in this way are only a small subset of all solutions and this is why other approaches are necessary. In particular, it is not clear a priori how typical these explicit solutions are. At the same time a solution which is given explicitly in terms of an algebraic expression involving 
elementary functions may be less useful in understanding the nature of the dynamics than a qualitative analysis.

There are many heuristic approaches. One is to find a direct analogy between the equation being studied and the equations describing a simple mechanical system. A well-known example is the analogy between the time evolution of a homogeneous and isotropic cosmological model and the motion of a particle in a potential. Another procedure is to discard terms which are believed to be small in a particular situation and study the resulting simplified equation. One case frequently considered is that of small perturbations of an explicit solution. Here the stability of the original solution, and thus its physical relevance, is being tested. A related but more general approach is to try a particular ansatz and to see whether it is formally consistent. The strength of heuristic approaches is that they often furnish a wealth of detailed information. A problematic aspect is that of their reliability. The success of a perturbative analysis in obtaining valid results often depends sensitively on the intuition of the person carrying it out.

An attractive feature of numerical calculations of solutions of differential equations is that they are guaranteed to give results. These results may or may not be correct. Again we have a problem of reliability. Numerical investigations can provide a quick way of checking a conjecture and thus of helping to put a research project on the right track. In some situations it may turn out that there is no alternative to numerical calculations and then tests of the reliability of the results which are internal to the numerical calculations themselves are of particular importance. These may take the form of convergence tests or of the comparison of two quite different numerical calculations which are expected to give the same answer. A good illustration of the latter procedure is the work of Gundlach 34] which provides one of the most convincing pieces of evidence that certain aspects of critical phenomena in gravitational collapse are not just numerical artefacts. It should be borne in mind that certain dynamical features of solutions of partial (or even ordinary) differential equations might not be accesible to numerical calculation due to limitations in resolution.

The application of the theory of partial differential equations is what will be referred to as the 'analytical' approach in the following. Frequently in textbooks phrases are encountered such as 'no exact solutions are available and so we are forced to resort to approximate methods'. This sweeping judgement overlooks the value of qualitative information of the kind which can be obtained by the analytical approach. This article is concerned with explaining what the analytical approach has to offer and comparing it with the other approaches already mentioned. Where possible the fruitful interactions between the different ways of proceeding will be emphasized. The discussion is limited to the Einstein evolution equations and due to the limitations of what can be contained in one article the important topic of the constraints is hardly touched on here. It is the subject of the article of Isenberg in these proceedings.

In the analytical study of the Einstein equations (or other partial differential equations such as those of hydrodynamics) the concepts of existence theorems and well-posedness play important roles. Here only those aspects of these ideas 
which are required in the sequel will be discussed. More detailed information can be found in [32. The properties which make up the definition of well-posedness of the initial value problem for an evolution equation are:

- The existence of a solution corresponding to any initial datum appropriate to the problem at hand.

- The uniqueness of the solution for a fixed initial datum.

- The continuous dependence of the solutions on the data. (A small change in the intial datum leads to a small change in the corresponding solution.)

The realization that well-posedness is important goes back to Hadamard in the first half of the twentieth century and it apparently took some time for mathematicians to be convinced. Hadamard discusses the concept in his book [35].

Ordinary differential equations with regular coefficients always have a wellposed initial value problem. This is by no means true for partial differential equations. It can be hard to prove well-posedness then. (It should be noted in passing that if the coefficients of an ODE are more or less singular then well-posedness cannot be taken for granted.)

A complication in studying well-posedness for partial differential equations is that it depends on the function spaces chosen for the data and the solutions. Related to this is the question of the sense in which the mapping from data to solutions is supposed to be continuous. This depends on the topology of the function spaces used. The type of evolution equations of most importance in the study of the Einstein equations are hyperbolic equations. The standard spaces used in this context are the Sobolev spaces of $L^{2}$ type, i.e. the spaces of functions which are square integrable together with their derivatives up to a specified order $s$. This property defines the Sobolev space $H^{s}$. In the limit of large $s$ we obtain the $C^{\infty}$ (smooth) functions. Well-posedness in any of these spaces with $s$ sufficiently large implies that the solutions are controlled pointwise by the initial data. Another class of functions which often plays a role is that of analytic $\left(C^{\omega}\right)$ functions, i.e. those which can be expanded in a convergent power series about any point. It is relatively easy to obtain existence theorems when working in the class of analytic functions using the theorem of Cauchy-Kowaleskaya. Unfortunately this does not prove that the solution can be controlled pointwise by pointwise bounds on the data and finitely many of its derivatives. Another disadvantage of analytic functions in this context is their unique continuation property which means that relativistic causality is obscured 32. There is a class of spaces intermediate between analytic and smooth functions which are sometimes encountered. These are the Gevrey spaces. (See, for instance 440 for an account of spaces of this kind.) They share the disadvantage of the analytic functions that bounds on infinitely many derivatives of the data is required to estimate solutions. On the other hand they do not have the unique continuation property. 
The Einstein equations have the special properties that existence is only obtained for initial data satisfying the constraint equations and that uniqueness only holds up to diffeomorphism. The notion of continuous dependence of solutions on data must be correspondingly modified. For more details see [32].

The plan of the article is as follows. In section 2 various results concerning ordinary differential equations are discussed. The third section explains the results of Lars Andersson and the author on constructing general spacetimes with singularities of a particular type. It also describes other applications of Fuchsian techniques in general relativity. Section 4 is concerned with the relations between analytical results and numerical relativity. The paper concludes with some ideas about the future of the field.

\section{Ordinary differential equations}

Although the main concern of this paper is with applications of partial differential equations, it should not be thought that all problems in general relativity which require the analysis of ordinary differential equations have been solved. Moreover, examples concerning ordinary differential equations can serve to illustrate issues which are also relevant for partial differential equations.

An interesting example of the interaction between numerical and analytical methods is the case of the Bartnik-McKinnon solutions. These are static spherically symmetric solutions of the Einstein-Yang-Mills equations. The problem of finding solutions of this type can be formulated in terms of a system of ordinary differential equations in a radial variable. Solutions which are asymptotically flat were found numerically by Bartnik and McKinnon [4]. These came as a surprise to many people. In this way a numerical calculation led to a conceptual enrichment. Some time later an existence proof for these solutions was given; the first result is in [53]. Unfortunately it does not appear that this existence proof led to new physical insights. It does not tell us more about why the solutions exist. A later alternative approach [14] seems to be better in this respect. Another point of view, put forward by Corlette and Wald [22], also offers an explanation, although it has not been made into an existence proof for the Bartnik-McKinnon solutions. (In related problems existence can be obtained in that way.)

For spatially homogeneous spacetimes the Einstein evolution equations reduce to ordinary differential equations. The dynamics of the solutions is very complicated, as exemplified by solutions of Bianchi type IX, also known as the Mixmaster model. There is an extensive literature where the Mixmaster model is studied by heuristic and numerical means. This work led to a consensus on what the main characteristics of the Mixmaster dynamics were but until recently there were no rigorous proofs. This changed with the work of Ringström [49, [50] where the key qualitative statements, i.e. the presence of infinitely many oscillations in the approach to the singularity and the unboundedness of the curvature there in every solution except the Taub-NUT solution, were established. Thus finally, after thirty years, the analysis of the Mixmaster model 
came into the fold of rigorous mathematics. In the picture of singularities in general spacetimes due to Belinskii, Khalatnikov and Lifshitz (BKL) \&] the Mixmaster solutions serve as the model for the general case. For this reason the fact of having a mathematical understanding of this model acquires a special significance.

There have also been other significant developments in the area of the rigorous treatment of Bianchi models recently. Examples are the analysis of the expanding phase of models of type $\mathrm{VII}_{0}$ with perfect fluid by Wainwright et. al. [57], 422 and that of the expanding phase of Bianchi type VIII vacuum models by Ringström [51] The analytic investigation of spatially homogeneous spacetimes now appears to be reaching a certain maturity. At the same time there are still many interesting open problems in this area. For instance the spacetimes of Bianchi type $\mathrm{VI}_{-1 / 9}$, which are believed to exhibit Mixmaster-like behaviour, have not yet been analysed rigorously. (See 56 for more information on these models.) Also, while the work of Ringström provides a lot of knowledge about untilted Bianchi models with perfect fluids, models with tilted fluid have only been studied in special cases [36]. A similar remark applies to Bianchi models with collisionless matter as source [45], 48$]$.

Another interesting case where ordinary differential equations can be applied to general relativity is in the study of self-similar spherically symmetric spacetimes. A good example is the proof by Christodoulou [19] of the existence of spherically symmetric asymptotically flat solutions of the Einstein equations coupled to a massless scalar field which develop naked singularities. (His later proof [20] that these solutions are non-generic in the class of spherically symmetric solutions of course uses partial differential equations.) For other models less is known about self-similar solutions. The work of 15] and references therein provides a lot of information about this in the context of a convenient dynamical systems formulation but this has not yet been exploited to provide a full mathematical analysis of the problem.

\section{$3 \quad$ Fuchsian systems and spacetime singularities}

In [3] a class of solutions of the Einstein equations coupled to a massless scalar field was constructed which are very general and which have initial singularities which can be described in detail. They provide the strongest mathematical support so far for the heuristic analysis of spacetime singularities due to Belinskii, Khalatnikov and Lifshitz. Near the singularity in these spacetimes the evolution at different spatial points decouples, and the full evolution can be approximated by a solution of a system of ordinary differential equations, the velocity dominated system. The spatial coordinates play the role of parameters. It is worth to remember that the BKL analysis is an outgrowth of the earlier heuristic analysis of Lifshitz and Khalatnikov [39] which gave the wrong answer (generic absence of singularities) and was shown to be in error by the rigorous mathematical arguments of the singularity theorems.

The statements of BKL concerned spacetimes depending on the maximum 
number of free functions, and that is precisely the sense in which the solutions whose existence is proved in [3] are general. In fact the free functions in [3] are required to be $C^{\omega}$, a restriction which does not seem natural from a physical point of view. It would be desirable to replace $C^{\omega}$ by $C^{\infty}$ and, as will be discussed below, there are indications that this should be possible. Counting functions is a crude way of assessing the generality of a class of solutions of a partial differential equation. A better characterization of generality is given in terms of open sets of initial data. It should be shown that the class of solutions constructed include all solutions arising from a non-empty open set of initial data on a regular Cauchy surface. The possibilities of doing this will be described below.

The method used in [3] is that of Fuchsian equations. Fuchsian equations are a class of differential equations whose coefficients have singularities of a certain type. They can be used to construct singular solutions of equations with regular coefficients. Suppose that a regular system of equations, such as the Einsteinscalar field system, is given. If an asymptotic form for singular solutions of this system can be guessed an ansatz can be made where the solution $u$ of the original system is expressed in terms of an explicit singular function $u_{0}$ and a remainder $u_{1}$ supposed to be regular. In favourable cases writing the original equations in terms of $u_{1}$ leads to a Fuchsian system, for which theorems on the existence of regular solutions are available. In general, once the ansatz containing some free functions has been introduced, the remainder $u_{1}$ is determined uniquely.

In the case of the Einstein-scalar equations the ansatz for the singular part is made in terms of a solution of the velocity dominated system. Velocity dominated quantities are denoted by a left upper index zero. The starting point is a solution of the velocity-dominated constraints. This consists of tensors ${ }^{0} g_{a b}$, ${ }^{0} k_{b}^{a},{ }^{0} \phi$ and $\partial_{t}{ }^{0} \phi$ in three dimensions which satisfy the equations

$$
\begin{gathered}
-{ }^{0} k_{a b}{ }^{0} k^{a b}+\left(\operatorname{tr}^{0} k\right)^{2}=8 \pi\left(\partial_{t}{ }^{0} \phi\right)^{2} \\
\nabla^{a}\left({ }^{0} k_{a b}\right)-\nabla_{a}\left(\operatorname{tr}^{0} k\right)=-8 \pi \partial_{t}{ }^{0} \phi \nabla_{a}^{0} \phi
\end{gathered}
$$

In these equations raising and lowering of indices and covariant derivatives correspond to the metric ${ }^{0} g_{a b}$. This system of partial differential equations can be solved in analogy to the full Einstein constraints by using the conformal method. Now the velocity dominated evolution equations should be solved for this initial data. This can be done essentially explicitly, with the result that

$$
\begin{gathered}
{ }^{0} \phi(t, x)=A(x) \log t+B(x) \\
{ }^{0} k^{a}{ }_{b}(t, x)=K^{a}{ }_{b}(x) t^{-1}
\end{gathered}
$$

after which the velocity dominated metric can be obtained by solving the equation $\partial_{t}^{0} g_{a b}=-2^{0} g_{a c}{ }^{0} k_{b}^{c}$. This can be done using matrix exponentials.

The next step is to find a unique solution of the full Einstein-scalar field equations which approaches the given velocity dominated solution as $t \rightarrow 0$. This can be achieved by applying the Fuchsian theory, provided all the eigenvalues of $K$ are positive. The resulting solution satisfies

$$
{ }^{0} g^{a c} g_{c b}=\delta_{b}^{a}+o(1)
$$




$$
\begin{gathered}
k^{a}{ }_{b}={ }^{0} k^{a}{ }_{b}+o\left(t^{-1}\right) \\
\phi={ }^{0} \phi+o(1)
\end{gathered}
$$

Corresponding statements are obtained for space and time derivatives. With this information the properties of the singularity can be computed by straightforward algebra. Consider for example the curvature invariant $R_{\alpha \beta} R^{\alpha \beta}$. By the field equations this is equal up to a constant factor to $\left(\nabla_{\alpha} \phi \nabla^{\alpha} \phi\right)^{2}$ The latter quantity is equal to $A^{4} t^{-4}$ up to an error of lower order in $t$. As a consequence of the constraints $A$ can never vanish. Hence the square of the Ricci tensor blows up uniformly as $t$ tends to zero and the singularity is a curvature singularity.

Fuchsian techniques have been applied to a number of other problems in general relativity and have the potential to be applied to many more. In [3] results were obtained for the Einstein equations coupled to a stiff fluid which are closely analogous to those for a massless scalar field. In [47] these results were extended to the case of a scalar field with mass or potential. The mass and potential terms become negligible near the singularity and the velocity dominated system is identical to that obtained for a massless scalar field. While the results just mentioned are the only ones of this degree of generality obtained so far Fuchsian techniques have also been applied to various situations with symmetry.

The theorem on Fuchsian systems applied in [3] was proved in [38, where some background discussion of Fuchsian methods can be found. The theorem there concerned only the analytic case. It was applied in that paper to Gowdy spacetimes, which are by now a standard testbed for applying new analytic techniques in general relativity. In [46] the theorems on the construction of Gowdy spacetimes with prescribed singularities were generalized to the $C^{\infty}$ case. Some of the steps in the argument did not depend on the details of the example being considered and should be much more generally applicable. With luck it will be possible to use them to handle the $C^{\infty}$ case for the Einsteinscalar system without symmetry. Some obstacles which have to be overcome were listed in 46]. Going beyond this to the question of obtaining an open set of initial data without making symmetry assumptions, no case involving the Einstein equations has been handled yet. There is, however, a treatment of this kind by Kichenassamy [37] of the nonlinear wave equation $\nabla_{\alpha} \nabla^{\alpha} u=-e^{u}$ in Minkowski space. It was shown using the Nash-Moser theorem that there is an open set of initial data giving rise to singularities of a particular form. It is to be hoped that this technique can be extended to the Einstein equations. There are already two results covering open sets of initial data for inhomogeneous spacetimes with particular symmetries [21], 44].

In the case of spacetime singularities which show behaviour of Mixmaster type Fuchsian techniques cannot be expected to apply and we have no method of constructing general spacetimes with this type of singularity. In [3] the scalar field was necessary and the approach would not work for a vacuum spacetime. It is worthwhile looking at what goes wrong. If the scalar field is absent then it is like having a scalar field with $A=0$. In that case it follows from the velocity dominated Hamiltonian constraint that one of the eigenvalues of $K$ 
must be negative and the assumptions of the theorem cannot be satisfied. The significance of the sign condition on the eigenvalues of $K$ is that it allows the growth of the spatial curvature as $t$ tends to zero to be estimated, thus showing that it eventually has a negligible effect. It may be noted that it might be possible to handle some negative eigenvalues and hence some vacuum solutions in higher dimensions (spacetime dimension $\geq 11$ ) since the algebra works out differently [24].

There has recently been significant progress in understanding Mixmaster behaviour in inhomogeneous spacetimes by heuristic and numerical techniques. See for instance [58, [6], [7]. Putting the results of this work on a rigorous mathematical footing is an fascinating challenge for the analytical approach to general relativity.

The discussion in this section up to now has been from the point of view of the BKL picture of spacetime singularities. In the past decade, starting with 443 it has been suggested that the singularities of the kind studied by BKL, which are in some sense spacelike, are not sufficient to describe the singularities inside black holes. Instead another type of singularities, called weak null singularities, are supposed to play an important role. Until recently the evidence for this was essentially heuristic or numerical in nature. This left some room for scepticism and so it is important that there is now a rigorous result by Dafermos [25] showing the development of weak null singularities from regular initial data and the occurrence of the associated effect of mass inflation.

That this kind of mathematical confirmation is not superfluous is shown by the story of the supposed stability of Cauchy horizons in the presence of a positive cosmological constant. This was suggested in [41] and led to several papers coming to similar conclusions over a period of several years. However it turned out in the end 13] that these conclusions were incorrect. The original work had observed that one mechanism leading to the instability of Cauchy horizons did not seem to work in that context. It turns out, however, that a different mechanism does appear to work. This throws some light on the difference between heuristic arguments and rigorous ones. In a mathematical argument all objections to a particular statement must be effectively ruled out. This means that a mathematical approach to a given problem is often much harder than a heuristic one. Once, however, a mathematical argument has been successfully completed it rules out the presence of any other effect that was not thought of the first time around.

\section{Numerical relativity}

This section is concerned with the relation of the analytical approach to the Einstein equations to numerical relativity. We have already seen one kind of interaction, which is that numerical results suggest a theorem which is later proved. Now the other direction will be considered, i.e. the question whether analytical results can be of use to numerical relativists in their choice of methods.

A lot of numerical relativity has been done using what are often called the 
ADM equations. What is meant by this is the Einstein evolution equations written in $3+1$ form in terms of the induced metric and second fundamental form of a foliation with a fixed choice of lapse and shift. In fact these equations were in the literature long before the works of Arnowitt, Deser and Misner (cf. [26]). Despite this the term 'ADM equations' will be used in this now widespread sense in the following, whereby the lapse and shift are restricted to take the simplest form that they are identically one and identically zero respectively. There is also an ambiguity due to the fact that the evolution equations are only defined modulo the constraints. Here only the vacuum equations are considered and the condition that the spatial components of the spacetime Ricci tensor vanish is taken to define the evolution equations. In fact the definition of the second fundamental form will be substituted into its evolution equation to produce a second order equation for the metric of the form $\partial_{t}^{2} g_{a b}=-2 R_{a b}+\ldots$. Here only the terms containing second derivatives of the metric have been written out.

Is the Cauchy problem for the ADM equations as just defined well-posed in the space of $C^{\infty}$ functions or in Sobolev spaces? Apparently nobody knows. It is known that there is a smooth solution of the ADM equations corresponding to any smooth initial datum satisfying the constraints and that the solutions depend continuously on the initial data. This is proved indirectly, for instance by the use of harmonic coordinates. This is all that is needed for the analytic theory of the Einstein equations. In numerical relativity, however, it cannot be expected that the initial data satisfy the constraints exactly. Thus it is of interest to know whether the ADM equations are well-posed as a system of evolution equations, independent of whether the constraints are satisfied or not. It has been shown by Choquet-Bruhat [17] that they are well-posed in Gevrey spaces, but the corresponding question for functions which are merely smooth, or belong to a Sobolev space, is open.

How can it be shown that a system of evolution equations is well-posed? This is related to the notion of hyperbolicity, which is at least intuitively equivalent to well-posedness of the initial value problem for appropriate initial data. It is perhaps useful to start by looking at the notion of ellipticity. Suppose that a nonlinear system of partial differential equations is given. Its ellipticity can be determined as follows. The system is elliptic if and only if its linearization about any given solution is elliptic. With this rule it then remains to define when a linear system is elliptic. To do this take the principal part (the part containing the derivatives of the highest order) and replace each derivative $\partial_{i}$ by $\xi_{i}$. This gives a homogeneous matrix-valued polynomial in $\xi$, called the principal symbol. The system is called elliptic if the principal symbol is invertible in the sense that for any $\xi \neq 0$ the values of the principal symbol are invertible matrices. More generally the set of non-zero $\xi$ for which the symbol is not invertible is called the characteristic set. Thus a system is elliptic if the characteristic set of its linearization is always empty.

Analogously to the elliptic case a non-linear system can be defined to be hyperbolic if its linearization about any solution is hyperbolic. Then it remains to define hyperbolicity in the linear case. There are criteria for hyperbolicity in terms of the principal symbol, but unfortunately, in contrast to the elliptic case, 
there is no simple and general condition. The characteristics are given as the roots of the characteristic polynomial, which is the determinant of the principal symbol. In general, for a system of $k$ equations of order $n$ the characteristic set has $k n$ sheets in the complex domain, counting multiplicity. If a system is hyperbolic then there must be $k n$ real sheets. In this case it is said that all characteristics are real. It was shown by Friedrich [30] that all the characteristics of the ADM equations are real. This is a positive sign but unfortunately does not suffice to show that the equations are hyperbolic.

Note that in order to check that a system is hyperbolic it does not suffice to verify that the Cauchy problems for all one-dimensional reductions are wellposed. An example of this is given in [23]. This equation is

$$
u_{t t t t}-2 u_{t t x x}-3 u_{t t y y}+u_{x x x x}+3 u_{x x y y}+2 u_{y y y y}+u_{x x y}=0
$$

It has an ill-posed Cauchy problem. Now consider solutions of the special form $u(t, x, y)=\tilde{u}(t, x \cos \alpha+y \sin \alpha)$. For each $\alpha$ this reduces the original equation to an equation for $\tilde{u}$ in one space dimension. This reduced equation has a well-posed Cauchy problem for each fixed value of $\alpha$. An important feature of this system is that the characteristics are of variable multiplicity. This example concerns a single higher order equation but presumably similar examples can be constructed for systems of first order equations.

To understand some of the issues involved in well-poseness it is useful to consider the trivial equation $u_{t t}=0$ for a function $u(t, x)$ of two variables. With initial data $u(t, 0)=f(0)$ and $u_{t}(t, 0)=g(x)$ the solution is $u(t, x)=$ $f(x)+\operatorname{tg}(x)$. If the equation were hyperbolic, like the wave equation, it would be natural to take $f$ in the Sobolev space $H^{s}$ and $g$ in $H^{s-1}$. Then the restriction of the solution to any other hypersurface of constant $t$ would be in $H^{s}$. However the explicit solution above is only in $H^{s-1}$ at later times. The equation is hyperbolic, but in a weaker sense than the wave equation since it loses one derivative. What is worse is that the hyperbolicity can be destroyed by adding lower order terms. The equation $u_{t t}=u_{x}$ (sideways heat equation) is not hyperbolic. It is not possible to bound the $H^{s}$ norm at a given time $t_{0}>0$ in terms of Sobolev norms of the initial data. This can be seen by doing a Fourier transform in $x$.

If the ADM equations are linearized about flat space the result has some similarity to the equation $u_{t t}=0$ and is weakly hyperbolic. If the full ADM equations are well-posed then it is reasonable to expect that their linearization about any background will have the same property. Some examples of linearization about simple non-trivial backgrounds will now be considered. The equations bear some resemblance to the equation $u_{t t}=u_{x}$ above. This approach has so far led to no evidence for ill-posedness of the ADM equations but it has not been carried very far. Perhaps a different choice of background would lead to a different result. Some possible directions to pursue will now be indicated.

In the examples to be considered here the metric can be written in the form

$$
d s^{2}=-d t^{2}+e^{2 \alpha(t, x)} d x^{2}+e^{2 \beta(t, x)} d y^{2}+e^{2 \gamma(t, x)} d z^{2}
$$


Ideally we would like a background metric such that the linearized Einstein equations have constant coefficients. Then taking a Fourier transform in space and time reduces the study of the growth of linearized solutions in time to an algebraic problem. It is natural to seek solutions with this property among spatially homogeneous solutions. As a first example, consider the Milne model which is given by ([56], p. 194):

$$
d s^{2}=-d t^{2}+t^{2}\left(d x^{2}+e^{2 x}\left(d y^{2}+d z^{2}\right)\right)
$$

This is of the form (9) with $\alpha=\log t$ and $\beta=\gamma=\log t+x$. Linearizing about this solution and denoting the linearized quantities corresponding to $\alpha, \beta$ and $\gamma$ by $\hat{\alpha}, \hat{\beta}$ and $\hat{\gamma}$ respectively leads to a system with coefficients depending explicitly on $t$. However this dependence on $t$ is sufficiently simple that introducing a new time coordinate by $\tau=\log t$ reduces the linearized Einstein equations to a system with constant coefficients. A special case consistent with the equations is obtained by setting $\beta=\gamma$. With $D=\partial / \partial \tau$ and ${ }^{\prime}=\partial / \partial x$ the system becomes

$$
\begin{aligned}
& -D^{2} \hat{\alpha}=3 D \hat{\alpha}+2 D \hat{\beta}+2 \hat{\alpha}^{\prime}+4 \hat{\alpha}-2 \hat{\beta}^{\prime \prime}-4 \hat{\beta}^{\prime} \\
& -D^{2} \hat{\beta}=D \hat{\alpha}+4 D \hat{\beta}+\hat{\alpha}^{\prime}+4 \hat{\alpha}-\hat{\beta}^{\prime \prime}-4 \hat{\beta}^{\prime}
\end{aligned}
$$

Note that while the equation for $\hat{\beta}$ contains the second spatial derivative of $\hat{\beta}$ the equation for $\hat{\alpha}$ does not contain the second spatial derivative of $\hat{\alpha}$ but does contain its first spatial derivative. This is a reason to suspect that wellposedness might fail as a consequence of the model equation discussed above. Now the equation will be Fourier transformed in space and time. Let $\omega$ and $\xi$ be Fourier variables corresponding to $\tau$ and $x$ respectively. Looking for solutions of the form $e^{i(\omega \tau+\xi x)}$ leads to consideration of the roots of the cubic equation for $\omega$ with coefficients depending on $\xi$

$$
\omega^{3}-7 i \omega^{2}+\left(-\xi^{2}+2 i \xi-14\right) \omega+\left(i \xi^{2}+2 \xi+8 i\right)=0
$$

By inspection $\omega=i$ is a root. It then only remains to solve a quadratic equation for $\omega$ to get the other two roots. These given by $\omega=2 i+\xi$ and $\omega=4 i-\xi$. The question of interest for well-posedness is whether the imaginary parts of the roots of the cubic equation for $\omega$ are bounded functions of $\xi$. In this example this is the case and no evidence for ill-posedness is obtained.

Consider next the Joseph solution, which is given by ([56], p. 197):

$$
\sinh 2 \tau\left[-d \tau^{2}+d x^{2}+A(\tau) e^{2 x} d y^{2}+A^{-1}(\tau) e^{2 x} d z^{2}\right]
$$

where $A(\tau)=(\tanh t)^{\sqrt{3}}$. Introduce a new time coordinate $t$ by means of the relation $d t=(\sinh 2 \tau)^{1 / 2} d \tau$. Then the form (9) can be obtained by setting $\sinh 2 \tau=e^{2 \alpha(t)}, A(\tau) e^{2 x} \sinh 2 \tau=e^{2 \beta(t, x)}$ and $A^{-1}(\tau) e^{2 x} \sinh 2 \tau=e^{2 \gamma(t, x)}$. In this case the coefficients of the linearized equations depend on $t$, although the dependence is not too complicated. One way to try to get an idea about wellposedness is to freeze the coefficients at a fixed time $t$ and do a Fourier tranform of the resulting system with constant coefficients. This has not been carried 
out. The conclusions to be drawn from these examples is that there are many calculations which might be done which could lead to a better understanding of the well-posedness or otherwise of the ADM equations. If the system is illposed, one single example could be the basis for a proof of ill-posedness of the full system. Unfortunately it not clear at this time what is the best place to look for an example of this kind.

Since a failure of well-posedness means a failure of continuous dependence of the solutions on the initial data and the initial data in a numerical problem are never exact the significance of results obtained using a numerical calculation with an ill-posed system is quite unclear. On the other hand it is known that a discretization of a partial differential equation may lead to a scheme which approximates a different equation better than the original one. (A well-known example of this is the introduction of numerical viscosity leading to a good approximation to a parabolic equation.) The ramifications of the well-posedness or otherwise of the ADM equations are still rather unclear.

In numerical relativity it is not just the well-posedness of the pure initial value problem which is important. It is necessary to impose boundary conditions and this leads to new difficulties. Friedrich and Nagy [31] have proved the wellposedness of certain initial boundary value problems for the (vacuum) Einstein equations but much more remains to be done. (See also [55] for some further recent work on the subject.)

An interesting puzzle on the interface between numerical and analytical work is provided by the BSSN system [52], [5]. It is apparently much better for numerical calculations than the ADM equations in some situations. It is not known to be hyperbolic although certain deformations of it were shown to give rise to symmetric hyperbolic systems in [32 and 33]. Different possible explanations of its superiority are discussed in [1] and [54]

\section{Outlook}

This section collects a few comments on some research directions which may become important in the near future, without any claim to completeness.

A topic which can be expected to play a major role in the applications of partial differential equations to general relativity in the future is that of the global stability of important explicit solutions. Until recently the only results of this kind were the theorems of Friedrich [29] on the stability of de Sitter space and Christodoulou and Klainerman [18] on the stability of Minkowski space. Now there are new developments in this area. Andersson and Moncrief have proved the stability of a vacuum cosmological model (the Milne model) in the expanding direction (see [2]) while Choquet-Bruhat and Moncrief [16]. have obtained a similar result for a vacuum cosmological model of Bianchi type III under the restriction that the perturbations are assumed to have one Killing vector. The Killing vector helps, but the Milne model is easier in other ways. It should be possible to generalize these results in many ways (different backgrounds and matter models). 
The results of 3 discussed in section 3 point the way to the possibility of proving the stability of the singularity in Friedmann models with a scalar field. Combining this with the results on the expanding phase mentioned above could lead to theorems on the global stability of open Friedmann models with suitable matter content. The stability of the singularity alone should allow conclusions about the stability of closed Friedmann models.

A subject of capital interest is that of the stability of the Schwarzschild and Kerr black holes. No nonlinear results are available at present although there has been recent progress concerning linear stability [9], [28]. The nonlinear stability of the Kerr solution is one of the outstanding open questions on the applications of partial differential equations to the Einstein equations.

The theme of critical phenomena has given rise to a considerable literature in general relativity. All of it is based on numerical calculations. The subject has been too tough to yield to analytical attack up to now. A promising approach to changing this unsatisfactory state of affairs is to study model problems. In fact it turns out that critical behaviour in gravitational collapse is not an isolated phenomenon within the field of partial differential equations. It is more likely to be typical of a kind of phenomenon which is widespread among solutions of nonlinear evolution equations. For instance Bizoń, Chmaj and Tabor 11 have studied wave maps in two space dimensions while Bizoń and Tabor [12] have studied the spherically symmetric Yang-Mills equations in space dimensions greater than three. It has not yet been possible to handle the dynamics analytically, but at least the critical solution itself has been proved to exist in one case 10.

The interaction between numerical and analytical work, some aspects of which were described in section 4 , has not really taken off, in the sense that a lot of practical computations in numerical relativity are little influenced by analytical developments. A notable exception is the numerical implementation of the conformal field equations, which has recently been reviewed by Frauendiener [27. It will be interesting to see whether this approach to numerical relativity eventually proves superior to more pedestrian methods.

\section{Acknowledgements}

I am grateful to Piotr Bizoń for discussions.

\section{References}

[1] Alcubierre, M., Allen, G., Brügmann, B., Seidel, E., Suen, W.-M. 2000 Towards an understanding of the stability properties of the $3+1$ evolution equations in general relativity. Phys. Rev. D62, 124011.

[2] Andersson, L. 1999 The global existence problem in general relativity. Preprint gr-qc/9911032. 
[3] Andersson, L., Rendall, A. D. 2001 Quiescent cosmological singularities. Commun. Math. Phys. 218, 479-511.

[4] Bartnik, R., McKinnon, J. 1988 Particle-like solutions of the Einstein-YangMills equations. Phys. Rev. Lett. 61, 141-144.

[5] Baumgarte, T., Shapiro, S. L. 1999 On the numerical integration of Einstein's field equations. Phys. Rev. D59, 024007.

[6] Berger, B. K., Moncrief, V. 1998 Evidence for an oscillatory singularity in generic U(1) symmetric cosmologies on $T^{3} \times R$. Phys. Rev. D, 064023 .

[7] Berger, B. K., Isenberg, J. and Weaver, M. 2001 Oscillatory approach to the singularity in vacuum spacetimes with $T^{2}$ isometry. Preprint grqc/0104048.

[8] Belinskii, V. A., Khalatnikov, I. M. and Lifshitz, E. M. 1982 A general solution of the Einstein equations with a time singularity. Adv. Phys. 31, 639-667.

[9] Beyer, H. 2000 On the stability of the Kerr metric. Preprint astro$\mathrm{ph} / 0008236$.

[10] Bizoń, P. 1999 Equivariant self-similar wave maps from Minkowski spacetime into 3-sphere. Preprint gr-qc/9910026.

[11] Bizoń, P., Chmaj, T. and Tabor, Z. 2000 Dispersion and collapse of wave maps. Nonlinearity 13, 1411-1423.

[12] Bizoń, P., Tabor, Z. 2001 On blowup for Yang-Mills fields. Preprint math$\mathrm{ph} / 0105016$.

[13] Brady, P. R., Moss, I. G. and Myers, R. C. 1998 Cosmic censorship: as strong as ever. Phys. Rev. Lett. 80, 3432-3435.

[14] Breitenlohner, P., Forgacs, P. and Maison, D. 1994 Static spherically symmetric solutions of the Einstein-Yang-Mills equations. Commun. Math. Phys. 163, 141-172.

[15] Carr, B. J., Coley, A. A., Goliath, M., Nilsson, U. S. and Uggla, C. 2000 The state space and physical interpretation of self-similiar spherically symmetric perfect-fluid solutions. Class. Quantum Grav. 18, 303-324.

[16] Choquet-Bruhat, Y., Moncrief, V. 2001 Future complete Einsteinian space times with $U(1)$ isometry group. C. R. Acad. Sci. 322, 137-144.

[17] Choquet-Bruhat, Y. To appear.

[18] Christodoulou, D., Klainerman, S. 1993 The global nonlinear stability of the Minkowski space. Princeton University Press, Princeton. 
[19] Christodoulou, D. 1994 Examples of naked singularity formation in the gravitational collapse of a scalar field. Ann. Math. 140, 607-653.

[20] Christodoulou, D. 1999 The instability of naked singularities in the gravitational collapse of a scalar field. Ann. Math. 149, 183-217.

[21] Chruściel, P. T. 1991 On uniqueness in the large of solutions of Einstein's equations (Strong Cosmic Censorship). Proceedings of the CMA Vol. 27. Australian National University.

[22] Corlette, K, Wald, R. 2001 Morse theory and infinite families of harmonic maps between spheres. Commun. Math. Phys. 215, 591-608.

[23] Courant, R. and Lax, A. 1955 Remarks on Cauchy's problem for hyperbolic partial differential equations with constant coefficients in several independent variables. Commun. Pure Appl. Math. 8, 497-502.

[24] Demaret, J, Henneaux, M. and Spindel, P. 1985 Non-oscillatory behaviour in vacuum Kaluza-Klein cosmologies. Phys. Lett. B 164, 27-30.

[25] Dafermos, M. 2001 Stability and instability of the Cauchy horizon for the spherically symmetric Einstein-Maxwell-scalar field equations. Preprint

[26] Fourès-Bruhat, Y. 1948 Sur l'intégration du probléme des conditions initiales en mécanique relativiste. C. R. Acad. Sci. (Paris) 226, 1071-1073.

[27] Frauendiener, J. 2000 Conformal infinity. Living Reviews in Relativity, article 2000-4.

[28] Friedman, J. and Morris, M. S. 2000 Schwarzschild perturbations die in time. J. Math. Phys. 41, 7529-7534.

[29] Friedrich, H. 1986 Existence and structure of past asymptotically simple solutions of Einstein's field equations with positive cosmological constant. J. Geom. Phys. 3, 101-117.

[30] Friedrich, H. 1996 Hyperbolic reductions for Einstein's equations. Class. Quantum Grav. 13, 1451-1469.

[31] Friedrich, H., Nagy, G. 1999 The initial boundary value problem for Einstein's vacuum field equations. Commun. Math. Phys. 201, 619-655.

[32] Friedrich, H., Rendall, A. D. 2000 The Cauchy problem for the Einstein equations. In B. G. Schmidt (ed) Einstein's Field Equations and Their Physical Implications. Lecture Notes in Physics 540. Springer, Berlin.

[33] Frittelli, S., Reula, O. 1999 Well-posed forms of the 3+1 conformallydecomposed Einstein equations. J. Math. Phys. 40, 5143-5156.

[34] Gundlach, C. 1995 The Choptuik spacetime as an eigenvalue problem. Phys. Rev. Lett. 75, 3214-3217. 
[35] Hadamard, J. 1952 Lectures on Cauchy's problem in linear partial differential equations. Dover, New York.

[36] Hewitt, C. G., Bridson, R. and Wainwright, J. 2001 The asymptotic regimes of tilted Bianchi II cosmologies. Gen. Rel. Grav. 33, 65-94.

[37] Kichenassamy, S. 1996 The blow-up problem for exponential nonlinearities. Commun. Part. Diff. Eq. 21, 125-162.

[38] Kichenassamy, S, Rendall, A. D. 1998 Analytic description of singularities in Gowdy spacetimes. Class. Quantum Grav. 15, 1339-1355.

[39] Lifshitz, E. M. and Khalatnikov, I. M. 1963 Investigations in relativistic cosmology. Adv. Phys. 12, 185-249.

[40] Mascarelli, M., Rodino, L. 1997 Partial differential equations with multiple characteristics. Akademie Verlag, Berlin.

[41] Mellor, F., Moss, I. G. 1990 Stability of black holes in de Sitter space. Phys. Rev. D41, 403-409.

[42] Nilsson, U. S., Hancock, M. J. and Wainwright, J. 2000 Non-tilted Bianchi $\mathrm{VII}_{0}$ models - the radiation fluid. Class. Quantum Grav. 17, 3119-3134.

[43] Poisson, E., Israel, W. 1990 Internal structure of black holes. Phys. Rev. D41, 1796-1809.

[44] Rein, G. 1996 Cosmological solutions of the Vlasov-Einstein system with spherical, plane or hyperbolic symmetry. Math. Proc. Camb. Phil. Soc. 119, 739-762.

[45] Rendall, A. D., Tod, K. P. 1999 Dynamics of spatially homogeneous solutions of the Einstein-Vlasov equations which are locally rotationally symmetric. Class. Quantum Grav. 16, 1705-1726.

[46] Rendall, A. D. 2000 Fuchsian analysis of singularities in Gowdy spacetimes beyond analyticity. Class. Quantum Grav. 17, 3305-3316.

[47] Rendall, A. D. 2000 Blow-up for solutions of hyperbolic PDE and spacetime singularities. Proceedings of Journées Equations aux Derivées Partielles, (Eds.) N. Depauw, D. Robert, X. Saint-Raymond. Groupement de Recherche 1151 du CNRS, 2000, XIV, 1-12.

[48] Rendall, A. D., Uggla, C. 2000 Dynamics of spatially homogeneous locally rotationally symmetric solutions of the Einstein-Vlasov equations. Class. Quantum Grav. 17, 4697-4714.

[49] Ringström, H. 2000 Curvature blow up in Bianchi VIII and IX vacuum spacetimes. Class. Quantum Grav. 17, 713-731.

[50] Ringström, H. 2000 The Bianchi IX attractor. Ann. H. Poincaré 2, 405-500. 
[51] Ringström, H. 2001 The future asymptotics of Bianchi VIII vacuum solutions. Preprint gr-qc/0103107.

[52] Shibata, M., Nakamura, T. 1995 Evolution of 3-dimensional gravitational waves - harmonic slicing case. Phys. Rev. D52, 5428-5444.

[53] Smoller, J., Wasserman, A., Yau, S.-T. and McLeod, J. 1991 Smooth static solutions of the Einstein-Yang-Mills equations. Commun. Math. Phys. 143, 115-147.

[54] Szilagyi, B., Gomez, R., Bishop, N. T. and Winicour, J. Cauchy boundaries in linearized gravitational theory. 1999 Phys. Rev. D62, 104006.

[55] Szilagyi, B., Schmidt, B., Winicour, J. 2001 Boundary conditions in linearized harmonic gravity. Preprint gr-qc/0106026.

[56] Wainwright, J., Ellis, G. F. R. 1997 Dynamical systems in cosmology. Cambridge University Press, Cambridge.

[57] Wainwright, J., Hancock, M. J. and Uggla, C. 1999 Asymptotic selfsimilarity breaking at late times in cosmology. Class. Quantum Grav. 16, 2577-2598.

[58] Weaver, M., Isenberg, J., Berger, B. K. 1998 Mixmaster behavior in inhomogeneous cosmological spacetimes Phys.Rev. Lett. 80, 2984-2987. 